\title{
smpr3d: an open-source toolkit for 3D phase-contrast imaging from 4D-STEM datasets
}

Philipp Pelz ${ }^{1}$, Hamish Brown ${ }^{2}$, Peter Ercius ${ }^{3}$, Ian Johnson ${ }^{3}$, Jim Ciston $^{4}$, Mary Scott $^{1}$ and Colin Ophus ${ }^{5}$

${ }^{1}$ UC Berkeley, Berkeley, California, United States, ${ }^{2}$ The University of Melbourne, Parkville, Victoria, Australia, ${ }^{3}$ Lawrence Berkeley National Laboratory, United States, ${ }^{4}$ UC Berkeley, California, United States, ${ }^{5}$ National Center for Electron Microscopy, Berkeley, California, United States

Most current phase-contrast reconstruction algorithms for 4D-STEM datasets, like differential phase contrast [1] and ptychography [2], assume and reconstruct a 2-dimensional image. Yet modern electron microscopes allow aberration-corrected imaging with numerical apertures that enable axial resolution on the sub-10nm scale, such that three-dimensional information is available from a single view for most samples of interest.

Reconstruction algorithms that allow to access this 3D phase-contrast information include multi-slice ptychography [6,7] and S-matrix phase-retrieval [3] and depth-sectioning [4,5]. Both algorithms have only recently been demonstrated for samples thicker than 2 depths of focus [4, 6], and are currently not widely available to the community.

In this talk we introduce smpr3d (pronounced "semper 3d", which stands for S-Matrix Phase Retrieval and 3D imaging; semper is latin for "always", meaning there is (almost) always 3D information in your data), an open-source toolkit implemented using python and pytorch, that allows reconstruction of 3D phase-contrast images from single 4D-STEM scans and 4D-STEM focal-series measurements, both on single commodity hardware accelerators and High-Performance Computing architectures.

We discuss common experimental parameters and preprocessing steps to produce 3D phase-contrast volumes from 4D-STEM measurements at the atomic scale.

Fig. 1 shows the usual workflow for reconstructing phase-contrast images from a single or multiple 4D-STEM scans. Given your microscope's chromatic aberrations and aberration correction capabilities, the optimal convergence angle and real-space sampling can be determined. After the defocus is accurately calibrated, 4DSTEM experiments can be performed, and 4D-STEM data preprocessed. S-matrix reconstruction yields a set of exit waves from all different incidence angles in the convergence aperture, which can then be used to synthesize virtual confocal phase images at arbitrary planes of the specimen in a last step.

Fig. 2 shows two atomic-resolution images of a complex carbon-nanotube sample obtained from a 4D-STEM defocal series with the 4Dcamera. smpr3d allows virtual refocusing along the beam direction, while the Smatrix reconstruction fully models multiple-scattering effects.

We will also demonstrate other virtual depth-sectioning modes enabled by the S-matrix reconstruction. [8] 


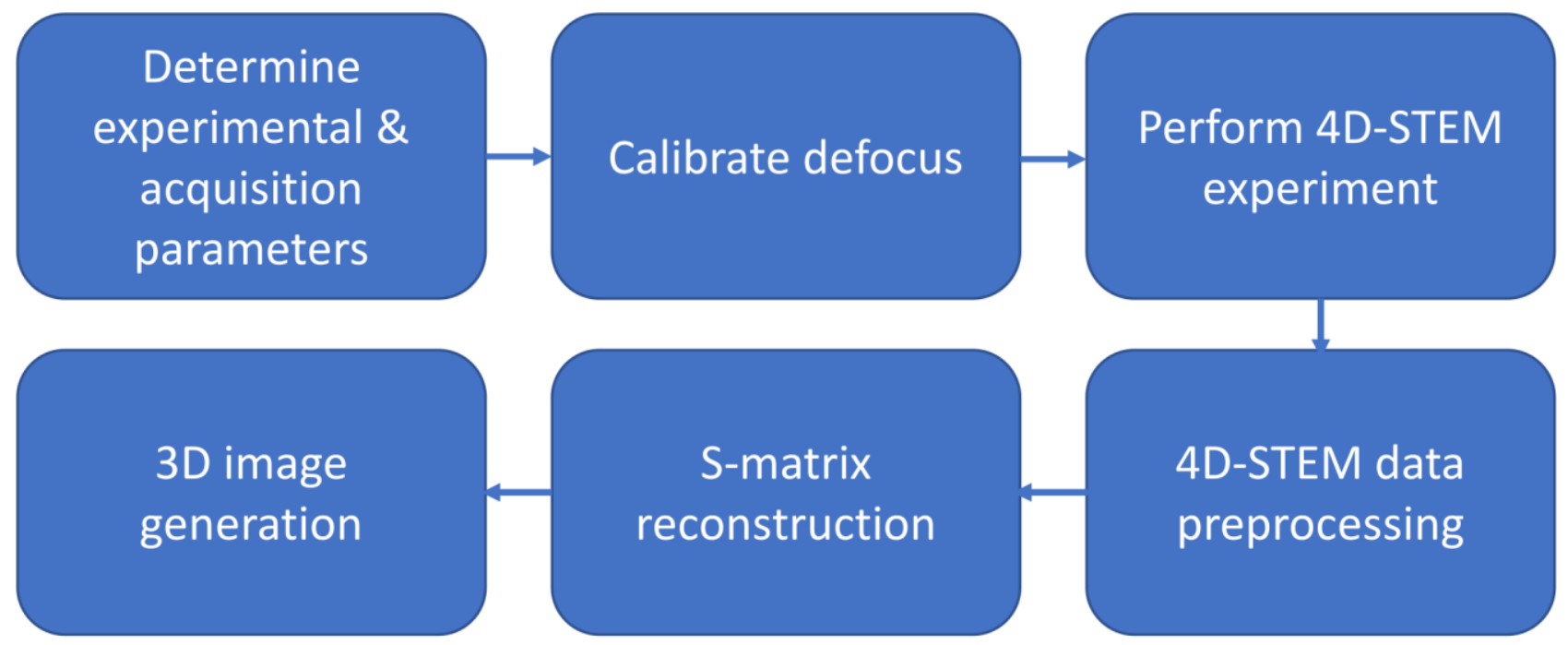

Figure 1. Fig. 1: Workflow for 3D phase reconstruction from 4D-STEM data.
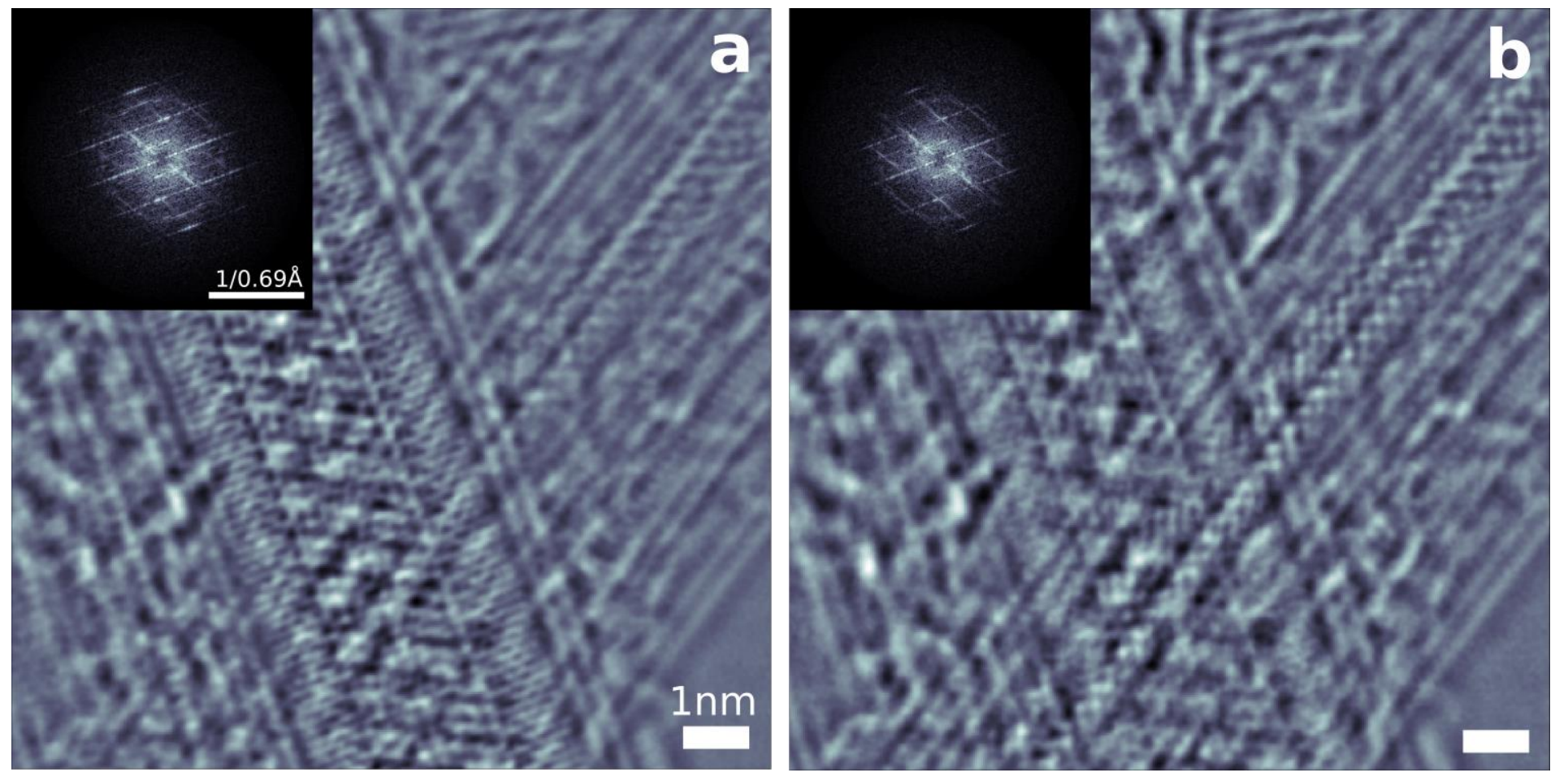

Figure 2. Fig. 2: Two z-slices through a complex carbon-nanotube sample generated via S-matrix depth sectioning from a 4D-STEM defocal series. a) phase-contrast image focused on the top nanotube at $\mathrm{z}=0$ with the power spectrum as inset. b) phase-contrast image focused at the bottom nanotubes at $\mathrm{z}=-9 \mathrm{~nm}$ with the power spectrum as inset.

References

[1] Shibata, N. et al. Differential phase-contrast microscopy at atomic resolution. Nature Physics 8, 611-615 (2012).

[2] Rodenburg, J. M. \& Bates, R. H. T. The Theory of Super-Resolution Electron Microscopy Via WignerDistribution Deconvolution. Philos. Trans. R. Soc. A Math. Phys. Eng. Sci. 339, 521-553 (1992). 
[3] Pelz, P. M. et al. Reconstructing the Scattering Matrix from Scanning Electron Diffraction Measurements Alone. (2020).

[4] Brown, H. G. et al. A three-dimensional reconstruction algorithm for scanning transmission electron microscopy data from thick samples. arXiv:2011.07652 [cond-mat] (2020).

[5] Ophus, C. et al. Advanced Phase Reconstruction Methods Enabled by Four-Dimensional Scanning Transmission Electron Microscopy. Microscopy and Microanalysis 25, 10-11 (2019).

[6] Gao, S. et al. Electron ptychographic microscopy for three-dimensional imaging. Nature Communications 8, (2017).

[7] Chen, Z. et al. Electron ptychography achieves atomic-resolution limits set by lattice vibrations. arXiv:2101.00465 [cond-mat] (2021).

[8] Work at the Molecular Foundry was supported by the Office of Science, Office of Basic Energy Sciences, of the U.S. Department of Energy under Contract No. DE-AC02-05CH11231. CO acknowledges additional support from the U.S. Department of Energy Early Career Research Program. HGB and JC acknowledge additional support from the Presidential Early Career Award for Scientists and Engineers (PECASE) through the U.S. Department of Energy. M. S. and P. P. acknowledge support from the STROBE NSF Science and Technology Center on Real-Time Functional Imaging. 